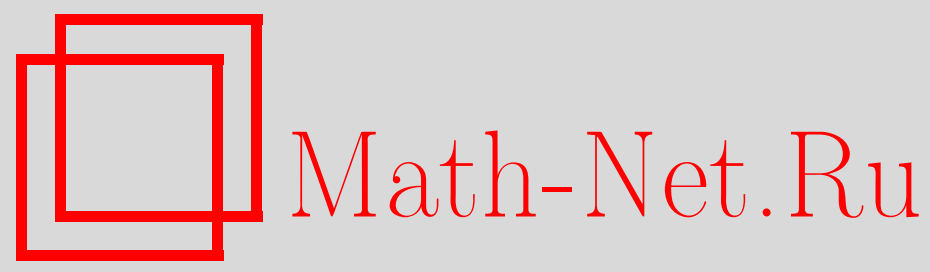

А. С. Тихомиров, О бирациональных преобразованиях схем Гильберта алгебраической поверхности, Матем. заметки, 2003, том 73, выпуск 2, 281-294

DOI: https://doi.org/10.4213/mzm187

Использование Общероссийского математического портала Math-Net.Ru подразумевает, что вы прочитали и согласны с пользовательским соглашением http://www . mathnet.ru/rus/agreement

Параметры загрузки:

IP: 54.197 .130 .99

26 апреля 2023 г., 13:20:10

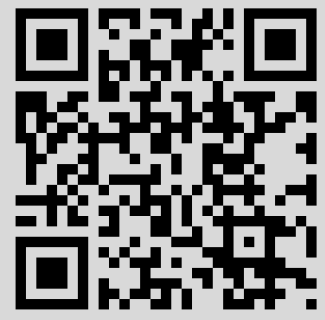




\section{О БИРАЦИОНАЛЬНЫХ ПРЕОБРАЗОВАНИЯХ СХЕМ ГИЛЬБЕРТА АЛГЕБРАИЧЕСКОЙ ПОВЕРХНОСТИ}

\section{А. С. Тихомиров}

В статье дается точное описание замыкания $\Gamma_{f}$ графики бирационального изоморфизма схем Гильберта точек алгебраических поверхностей $f: \mathrm{Hilb}^{d} \widetilde{S} \rightarrow \mathrm{Hilb}^{d} S$, соответствующего раздутию $\sigma: \widetilde{S} \rightarrow S$ с центром в точке на гладкой алгебраической поверхности $S$. Доказьвается, что проекция $\operatorname{pr}_{\widetilde{H}}: \Gamma_{f} \rightarrow \widetilde{H}=\operatorname{Hilb}^{d} \widetilde{S}$ есть раздутие с центром в подмногообразии инциденции $R \subset \widetilde{H}$, параметризующем наборы $d$ точек в $\widetilde{S}$, из которых по меньшей мере две точки инцидентны исключительной прямой раздутия $\sigma$; при этом $R$ снабжается схемной структурой посредством подходящего пучка идеалов Фиттинга. Доказывается, что $\Gamma_{f}$ неособо только при $d \leqslant 2$, и в нетривиальом случае $d=2$ дается точное описание разложения второй проекции $\operatorname{pr}_{H}: \Gamma_{f} \rightarrow H=\operatorname{Hilb}^{d} S$ в композицию двух раздутий с гладкими центрами.

Библиограффия: 8 названий.

Введение. Настоящая статья посвящена описанию бирациональных перестроек схем Гильберта точек гладкой алгебраической поверхности, индуцированных бирациональными преобразованиями поверхности. Поскольку бирациональные преобразования гладкой поверхности разлагаются в композицию раздутий точек ( $\sigma$-процессов) и обратных к ним преобразований стягивания, достаточно рассмотреть случай перестройки схемы Гильберта при $\sigma$-процессе. Пусть $S$ - гладкая алгебраическая поверхность над алгебраически замкнутым полем $k$. Рассмотрим схему Гильберта точек $H=H_{d}:=$ $\mathrm{Hilb}^{d} S$, параметризующую 0-мерные подсхемы длины $d$ в $S$ (системы точек). Как известно [1], $H$ - гладкое неприводимое $2 d$-мерное многообразие. Пусть $\sigma: \widetilde{S} \rightarrow S-$ раздутие с центром в (приведенной) замкнутой точке $x_{0} \in S, l_{0}:=\sigma^{-1}\left(x_{0}\right) \simeq \mathbf{P}^{1}$ - исключительная прямая на $\widetilde{S}$, и пусть $\widetilde{H}=\mathrm{Hilb}^{d} \widetilde{S}$. Раздутие $\sigma$ индуцирует бирациональньй изоморфизм

$$
f: \widetilde{H} \rightarrow H .
$$

Естественньм разрешением особенностей бирационального изоморфизма $f$ является замыкание $\Gamma_{f}$ графика отображения $f$ с регулярными проекциями $\widetilde{H} \stackrel{\mathrm{pr}_{\tilde{H}}}{\leftarrow} \Gamma_{f} \stackrel{\mathrm{pr}_{H}}{\rightarrow} H$, так что $\operatorname{pr}_{H}=f \circ \mathrm{pr}_{\tilde{H}}$. (Это разрешение минимально в обычном смысле: всякое многообразие $H^{\prime}$ с регулярными бирациональными проекциями $\widetilde{H} \stackrel{p_{1}}{\leftarrow} H^{\prime} \stackrel{p_{2}}{\rightarrow} H$ такими, что $p_{2}=f \circ p_{1}$, доминирует над $\Gamma_{f}$, т.е. существует морфизм $\phi: H^{\prime} \rightarrow \Gamma_{f}$ такой, что

Работа выполнена при частичной поддержке гранта INTAS-OPEN-2000-269. 
$p_{1}=\operatorname{pr}_{\tilde{H}} \circ \phi, p_{2}=\operatorname{pr}_{H} \circ \phi$. При этом $\Gamma_{f}$ определяется указанным свойством минимальности однозначно с точностью до изоморфизма.)

Наша цель - получение точного описания многообразия $\Gamma_{f}$ через бирациональньй морфизм - проекцию $\operatorname{pr}_{\widetilde{H}}: \Gamma_{f} \rightarrow \widetilde{H}$ как раздутие с центром в соответствующей подсхеме $R$ в $\widetilde{H}$. Первьй основной результат статьи - теорема 1.3 - показьвает, что $R$ как замкнутое подмножество в $\widetilde{H}$ есть цикл инциденции ${ }^{1}$

$$
R=\left\{z \in \widetilde{H} \mid \operatorname{length}\left(Z \cap l_{0}\right) \geqslant 2\right\},
$$

а схемная структура на $R$ задается пучком идеалов Фиттинга

$$
\operatorname{Fitt}^{0}\left(\operatorname{pr}_{2 *} R^{1}\left(\sigma \times \operatorname{id}_{\widetilde{H}}\right)_{*} \mathscr{J}_{\widetilde{\Gamma}, \widetilde{S} \times \widetilde{H}}\right)
$$

где

$$
\widetilde{\Gamma}=\{(x, z) \in \widetilde{S} \times \widetilde{H} \mid x \in Z\}
$$

- универсальньй цикл. Первьй нетривиальный случай здесь -это $d=2$. В этом случае вьшеприведенная структура на $R$ есть структура гладкого многообразия (см. замечание 1.1 ниже) и, тем самьм, $\Gamma_{f}$ - также гладкое многообразие. При этом второй основной результат работы - теорема 2.6 - дает точное описание второй проекции $\mathrm{pr}_{H}: \Gamma_{f} \rightarrow H$ в композицию двух раздутий с гладкими центрами.

В заключении статьи рассматривается общий случай $d \geqslant 3$. В этом случае, как показывает простая проверка, уже не только схема $R$ имеет особенности (хотя и приведена в общей точке), но и $\Gamma_{f}$ является особым многообразием (теорема 3.1 ). Таким образом, $\Gamma_{f}$ неособо только при $d \leqslant 2$.

1. Бирациональный изоморфизм схем Гильберта $\mathrm{Hilb}^{d} \widetilde{S} \rightarrow \mathrm{Hilb}^{d} S$, индуцированный $\sigma$-процессом $\widetilde{S} \rightarrow S$. Этот пункт посвящен описанию замькания $\Gamma_{f}$ графика бирационального отображения $f: \widetilde{H} \rightarrow H$ из (1). Рассмотрим композицию проекций

$$
\widetilde{S} \times \widetilde{H} \stackrel{\bar{\sigma}:=\sigma \times \mathrm{id}_{\widetilde{H}}}{\longrightarrow} S \times \widetilde{H} \stackrel{\mathrm{pr}_{2}}{\longrightarrow} \widetilde{H}
$$

и определяемьй ею пучок идеалов Фиттинга

$$
\mathbf{J}=\operatorname{Fitt}^{0}\left(\operatorname{pr}_{2 *} R^{1} \bar{\sigma}_{*} \mathscr{J}_{\widetilde{\Gamma}, \tilde{S} \times \tilde{H}}\right) .
$$

Заметим, что по свойству раздутия $\sigma$ имеют место равенства

$$
\bar{\sigma}_{*} \mathscr{O}_{\widetilde{S} \times \tilde{H}}=\mathscr{O}_{S \times \tilde{H}}, \quad R^{i} \bar{\sigma}_{*} \mathscr{O}_{\widetilde{S} \times \tilde{H}}=0, \quad i>0 .
$$

Покажем, что

$$
\operatorname{Supp}\left(\mathscr{O}_{\widetilde{H}} / \mathbf{J}\right)=R,
$$

где $R$ определено вьше во введении. Для этого рассмотрим в $S \times \widetilde{H}$ подмножество $\Gamma_{0}=$ $\bar{\sigma}(\widetilde{\Gamma})$ как приведенное неприводимое подмногообразие (напомним, что $\widetilde{\Gamma}$ неприводимо cм., например, [1]). Положим $\widetilde{\Gamma}_{R}=\widetilde{\Gamma} \cap\left\{l_{0}\right\} \times R$. Из определения множества $R$ непосредственно следует, что

(а) $\bar{\sigma} \mid \underset{\Gamma}{\widetilde{\Gamma}} \backslash \widetilde{\Gamma}_{R}: \widetilde{\Gamma} \backslash \widetilde{\Gamma}_{R} \rightarrow \Gamma_{0} \backslash\left\{x_{0}\right\} \times R$ - изоморфизм, а

(б) $\bar{\sigma} \mid \widetilde{\Gamma}_{R}: \widetilde{\Gamma}_{R} \rightarrow\left\{x_{0}\right\} \times R-$ конечньй морфизм степени $\geqslant 2$.

\footnotetext{
${ }^{1}$ Здесь и ниже мы пользуемся следующим стандартным соглашением об обозначениях: для произвольной точки $z \in H$ (соответственно $z \in \widetilde{H}$ ) через $Z$ будем обозначать соответствующую подсхему длины $d$ в $S$ (соответственно в $\widetilde{S})$.
} 
Таким образом, $\bar{\sigma} \mid \widetilde{\Gamma}: \widetilde{\Gamma} \rightarrow \Gamma_{0}-$ конечньй бирациональньй морфизм. Тем самым, ввиду неприводимости $\widetilde{\Gamma}$ пучок $\bar{\sigma}_{*} \mathscr{O}_{\widetilde{\Gamma}}$ является $\mathscr{O}_{\Gamma_{0}}$-пучком чистой размерности, равной $\operatorname{dim} \Gamma_{0}$ (т.е. не имеет подпучков кручения на $\Gamma_{0}$ ). Поэтому пучок $\bar{\sigma}_{*} \mathscr{J}_{\widetilde{\Gamma}}$ как подпучок в $\mathscr{O}_{S \times \tilde{H}}$ (здесь вложение $\bar{\sigma}_{*} \mathscr{J}_{\widetilde{\Gamma}, \widetilde{S} \times \widetilde{H}} \hookrightarrow \mathscr{O}_{S \times \widetilde{H}}$ является следствием первого равенства (4)) совпадает с пучком идеалов подмногообразия $\Gamma_{0}$ в $S \times \widetilde{H}$ :

$$
\bar{\sigma}_{*} \mathscr{J}_{\widetilde{\Gamma}, \widetilde{S} \times \tilde{H}}=\mathscr{J}_{\Gamma_{0}, S \times \widetilde{H}}
$$

Теперь применим функтор $R^{i} \bar{\sigma}_{*}$ к точной тройке

$$
0 \rightarrow \mathscr{J}_{\widetilde{\Gamma}, \widetilde{S} \times \widetilde{H}} \rightarrow \mathscr{O}_{\widetilde{S} \times \widetilde{H}} \rightarrow \mathscr{O}_{\widetilde{\Gamma}} \rightarrow 0
$$

с учетом (4) получим точную последовательность

$$
0 \rightarrow \bar{\sigma}_{*} \mathscr{J}_{\widetilde{\Gamma}, \tilde{S} \times \tilde{H}} \rightarrow \mathscr{O}_{S \times \tilde{H}} \rightarrow \bar{\sigma}_{*} \mathscr{O}_{\widetilde{\Gamma}} \rightarrow R^{1} \bar{\sigma}_{*} \mathscr{J}_{\widetilde{\Gamma}, \widetilde{S} \times \tilde{H}} \rightarrow 0
$$

которая ввиду (6) расщепляется на две точные тройки:

$$
\begin{gathered}
0 \rightarrow \mathscr{J}_{\Gamma_{0}, S \times \widetilde{H}} \rightarrow \mathscr{O}_{S \times \tilde{H}} \rightarrow \mathscr{O}_{\Gamma_{0}} \rightarrow 0, \\
0 \rightarrow \mathscr{O}_{\Gamma_{0}} \rightarrow \bar{\sigma}_{*} \mathscr{O}_{\widetilde{\Gamma}} \rightarrow R^{1} \bar{\sigma}_{*} \mathscr{J}_{\widetilde{\Gamma}, \tilde{S} \times \tilde{H}} \rightarrow 0 .
\end{gathered}
$$

Из тройки (8) и вышеуказанных свойств (а) и (б) морфизма $\bar{\sigma}$ непосредственно следует, что $\operatorname{Supp}\left(R^{1} \bar{\sigma}_{*} \mathscr{J}_{\widetilde{\Gamma}}, \widetilde{S} \times \widetilde{H}\right)=\left\{x_{0}\right\} \times R$, а значит, ввиду изоморфизма $\operatorname{pr}_{2} \mid\left\{x_{0}\right\} \times R$ : $\left\{x_{0}\right\} \times R \stackrel{\sim}{\rightarrow} R$,

$$
\operatorname{Supp}\left(\mathrm{pr}_{2 *} R^{1} \bar{\sigma}_{*} \mathscr{J}_{\widetilde{\Gamma}, \tilde{S} \times \tilde{H}}\right)=R,
$$

что по определению идеала Фиттинга Fitt $^{0}$ равносильно (5).

ЗАмЕчАниЕ 1.1 . В случае длины $d=2$ из определения $R$ непосредственно следует, что $R=\mathrm{Hilb}^{2} l_{0} \simeq S^{2} l_{0}-$ гладкое подмногообразие в $\widetilde{H}$, изоморфное $\mathbf{P}^{2}$, причем

$$
\sigma_{R}:=\bar{\sigma} \mid \widetilde{\Gamma}_{R}: \widetilde{\Gamma}_{R} \rightarrow\left\{x_{0}\right\} \times R \stackrel{\sim}{\rightarrow} \mathbf{P}^{2}
$$

есть двойное накрытие с ветвлением в диагональной конике $C \subset \mathbf{P}^{2}$, так что

$$
\sigma_{R *} \mathscr{O}_{\widetilde{\Gamma}_{R}} \simeq \mathscr{O}_{\mathbf{P}^{2}} \oplus \mathscr{O}_{\mathbf{P}^{2}}(-1)
$$

и, более того, с учетом свойств (а) и (б) многообразия $\widetilde{\Gamma}_{R}$ выше

$$
\bar{\sigma}_{*} \mathscr{O}_{\widetilde{\Gamma}} / \mathscr{O}_{\Gamma_{0}} \simeq \sigma_{R *} \mathscr{O}_{\widetilde{\Gamma}_{R}} / \mathscr{O}_{\mathbf{P}^{2}} \simeq \mathscr{O}_{\mathbf{P}^{2}}(-1)
$$

Тем самым, ввиду (8) и того, что $\mathrm{pr}_{2} \mid\left\{x_{0}\right\} \times R:\left\{x_{0}\right\} \times R \stackrel{\sim}{\rightarrow} R$ - изоморфизм, имеем $\operatorname{pr}_{2 *} R^{1} \bar{\sigma}_{*} \mathscr{J}_{\widetilde{\Gamma}, \widetilde{S} \times \widetilde{H}} \simeq \mathscr{O}_{\mathbf{P}^{2}}(-1)$, а значит, $\mathbf{J} \simeq \operatorname{Fitt}^{0}\left(\mathscr{O}_{\mathbf{P}^{2}}(-1)\right)$ совпадает с пучком идеалов $\mathscr{J}_{R, \widetilde{H}}$ гладкого подмногообразия $R$ в $\widetilde{H}$.

Рассмотрим раздутие $\delta: \widehat{H} \rightarrow \widetilde{H}$ с центром в подсхеме $R \subset \widetilde{H}$, определяемой идеалом J. Наша цель - доказать совпадение $\widehat{H}$ с $\Gamma_{f}$. Сначала докажем существование 
бирационального морфизма $\gamma: \widehat{H} \rightarrow H$ такого, что $f \circ \delta=\gamma$. Для этого рассмотрим расслоенные произведения $\widehat{\Gamma}_{0}=\Gamma_{0} \times_{\widetilde{H}} \widehat{H}$ и $\widehat{\Gamma}=\widetilde{\Gamma} \times_{\Gamma_{0}} \widehat{\Gamma}_{0}$ и образуемую ими диаграмму расслоенных квадратов, где $\sigma_{0}=\bar{\sigma} \mid \widetilde{\Gamma}$, а $q_{0}$ есть композиция $\Gamma_{0} \hookrightarrow S \times \widetilde{H} \stackrel{\mathrm{pr}_{2}}{\rightarrow} \widetilde{H}$ :

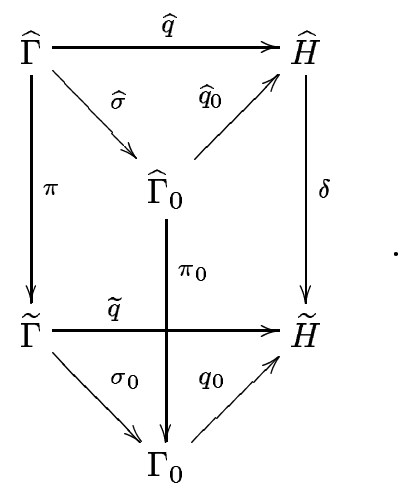

Так как по свойству универсального цикла $\widetilde{\Gamma}$ проекция $\tilde{q}: \widetilde{\Gamma} \rightarrow \widetilde{H}-$ плоский конечньй морфизм степени $d$, то пучок

$$
E=\tilde{q}_{*} \mathscr{O}_{\widetilde{\Gamma}}=q_{0 *} \sigma_{0 *} \mathscr{O}_{\widetilde{\Gamma}}
$$

является локально свободным $\mathscr{O}_{\widetilde{H}}$-пучком ранга $d$. Отсюда и из неприводимости многообразия $\widehat{H}$ (общее свойство раздутий) и неприводимости $\widetilde{\Gamma}$ следует, что в расслоенной диаграмме (10) схема $\widehat{\Gamma}$ - также неприводимое многообразие, $\hat{q}: \widehat{\Gamma} \rightarrow \widehat{H}-$ плоский конечньй морфизм. При этом замена базы показьвает, что

$$
\hat{q}_{*} \mathscr{O}_{\widehat{\Gamma}}=\delta^{*} E
$$

- локально свободньй $\mathscr{O}_{\widehat{H}}$-пучок ранга $d$. Кроме того, из диаграммы $(10)$ в силу конечности (а значит, аффинности) морфизмов $\hat{q}_{0}$ и $q_{0}$ также вытекают равенства

$$
\widehat{q}_{0 *} \mathscr{O}_{\widehat{\Gamma}_{0}}=\widehat{q}_{0 *} \pi_{0}^{*} \mathscr{O}_{\Gamma_{0}}=\delta^{*} q_{0 *} \mathscr{O}_{\Gamma_{0}} .
$$

Так как $q_{0}$ - конечный морфизм, то, применяя к тройке (8) функтор $R^{i} q_{0 *}$, с учетом (11) получаем

$$
0 \rightarrow q_{0 *} \mathscr{O}_{\Gamma_{0}} \rightarrow E \rightarrow F \rightarrow 0
$$

где

$$
F:=\operatorname{pr}_{2 *} R^{1} \bar{\sigma}_{*} \mathscr{J}_{\widetilde{\Gamma}, \widetilde{S} \times \widetilde{H}}
$$

Применим теперь к (14) функтор $\delta^{*}$; с учетом (13) получаем тройку

$$
0 \rightarrow \widehat{q}_{0 *} \mathscr{O}_{\widehat{\Gamma}_{0}} \stackrel{i}{\rightarrow} \delta^{*} E \rightarrow \delta^{*} F \rightarrow 0
$$

Эта тройка точна слева: в самом деле, ввиду неприводимости $\Gamma_{0}$ и того, что $\widehat{q}_{0}: \widehat{\Gamma}_{0} \rightarrow \widehat{H}-$ конечньй сюръективный морфизм, $\mathscr{O}_{\widehat{H}^{-}}$-пучок $\widehat{q}_{0 *} \mathscr{O}_{\widehat{\Gamma}_{0}}$ не имеет подпучков кручения, поэтому морфизм $i$ в (16), будучи по построению инъективным в общей точке, инъективен. Кроме того, в силу известных свойств раздутий [2, гл. 2, 7.13] (в нашем случае, т.е. в случае раздутия $\delta: \widehat{H} \rightarrow \widetilde{H})$, пучок

$$
\operatorname{Fitt}^{0}\left(\delta^{*} F\right)=\delta^{-1} \operatorname{Fitt}^{0}(F) \cdot \mathscr{O}_{\widehat{H}}=\delta^{-1} \mathbf{J} \cdot \mathscr{O}_{\widehat{H}}
$$

(здесь в первом равенстве мы пользуемся функториальностью идеалов Фиттинга) является обратимым $\mathscr{O}_{\widehat{H}}$-пучком.

Теперь воспользуемся следующим техническим результатом (см. [3, лемма 1]). 
Лемма 1.2. Пусть $X$ - челая нётерова схема и $F$ - ненулевой когерентный $\mathscr{O}_{X}$-пучок кручения. Тогда пучок идеалов фиттинга $\operatorname{Fitt}^{0}(F)$ является обратимым $\mathscr{O}_{X}$-пучком тогда и только тогда, когда $F$ имеет гомологическую размерность 1: $d h_{X} F=1$.

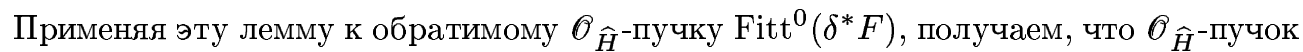
кручения $\delta^{*} F$ имеет гомологическую размерность 1 . Отсюда и из точной тройки (16) ввиду локальной свободы пучка $\delta^{*} E$ следует, что пучок $\widehat{q}_{0 *} \mathscr{O}_{\widehat{\Gamma}_{0}}$ локально свободен ранга $d$, что, в свою очередь, равносильно тому, что конечньй морфизм $\widehat{q}_{0}: \widehat{\Gamma}_{0} \rightarrow \widehat{H}$ является плоским морфизмом степени $d$ (см., например, [4, лекция 6, предложение 7]). Заметим, что по построению проекция $\widehat{q}_{0 *}$ разлагается в композицию $\widehat{\Gamma}_{0} \hookrightarrow S \times \widehat{H} \stackrel{\mathrm{pr}_{2}}{\rightarrow} \widehat{H}$. Тем самым, по универсальному свойству схемы Гильберта $H$ определен морфизм $\gamma: \widehat{H} \rightarrow H$ такой, что $\widehat{\Gamma}_{0}=\left(\gamma \times \operatorname{id}_{H}\right)^{-1}(\Gamma)$, где $\Gamma \subset S \times H-$ универсальный цикл. При этом по конструкции, очевидно, $\gamma=f \circ \delta$.

Теперь рассмотрим произвольное разрешение бирационального изоморфизма $f: \widetilde{H}$ $\rightarrow H$, т.е. неприводимое многообразие $H^{\prime}$ с бирациональньми морфизмами $\widetilde{H} \underset{1}{p_{1}} H^{\prime}$ $\stackrel{p_{2}}{\rightarrow} H$ такими, что $p_{2}=f \circ p_{1}$, и докажем существование морфизма $\phi: H^{\prime} \rightarrow \widehat{H}$, включающегося в коммутативную диаграмму

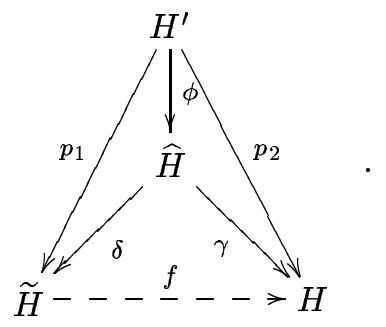

Отсюда и из свойства минимальности $\Gamma_{f}$ (см. введение) будет следовать, что $\Gamma_{f}=\widehat{H}$. Для доказательства существования морфизма $\phi$ рассмотрим сначала диаграмму

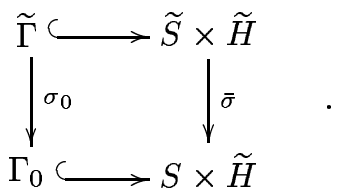

Так как $\bar{\sigma}$ - раздутие с центром $\left\{x_{0}\right\} \times \widetilde{H}$, а $\sigma_{0}$ - бирациональньй морфизм неприводимых многообразий, то диаграмма (19) является расслоенньм квадратом (в котором, тем самым, $\sigma_{0}$ - также раздутие с центром $\left.\Gamma_{0} \cap\left\{x_{0}\right\} \times \widetilde{H}\right)$. Далее, рассмотрим диаграмму 
расслоенных квадратов

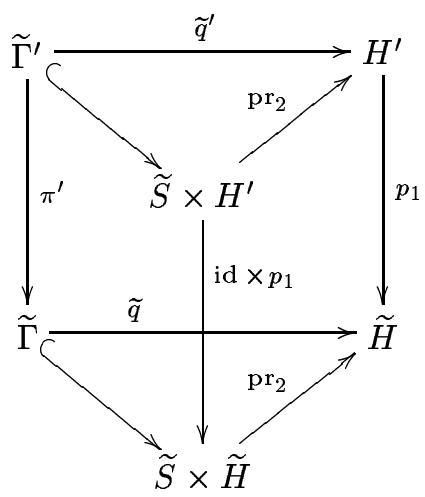

Так как $\tilde{q}$ и $\tilde{q}^{\prime}$ - плоские конечные морфизмы, а $p_{1}$ - бирациональный морфизм неприводимых многообразий, то, рассуждая как и выше с неприводимостью многообразия $\widehat{\Gamma}$, получаем, что $\widetilde{\Gamma}^{\prime}$ неприводимо. Аналогично, рассматривая многообразие $\Gamma^{\prime}=\Gamma \times_{H} H^{\prime}$ с проекцией $q^{\prime}: \Gamma^{\prime} \hookrightarrow S \times H^{\prime} \rightarrow H^{\prime}$, являющейся конечным плоским морфизмом степени $d$, получаем неприводимость $\Gamma^{\prime}$. Далее, по построению коммутативна диаграмма

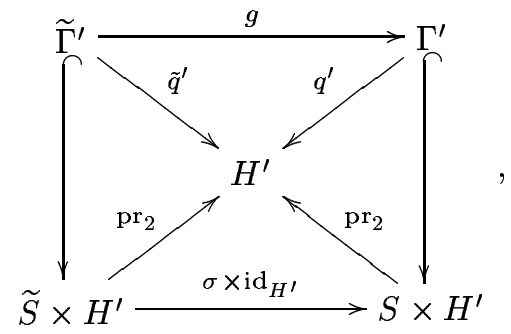

в которой $g:=\sigma \times \operatorname{id}_{H^{\prime}} \mid \widetilde{\Gamma}^{\prime}: \widetilde{\Gamma}^{\prime} \rightarrow \Gamma^{\prime}-$ сюръективный бирациональный морфизм ввиду неприводимости $\widetilde{\Gamma}^{\prime}$ и $\Gamma^{\prime}$. Отсюда, так как $\tilde{q}^{\prime}$ и $q^{\prime}$ - плоские конечные морфизмы степени $d$, получаем, что $\tilde{q}^{\prime}=q^{\prime} \circ g$ есть разложение Штейна морфизма $\tilde{q}^{\prime}([2$, гл. $3,11.5])$, так что $g: \widetilde{\Gamma}^{\prime} \rightarrow \Gamma^{\prime}-$ изоморфизм:

$$
g: \widetilde{\Gamma}^{\prime} \stackrel{\sim}{\rightarrow} \Gamma^{\prime}
$$

Теперь расслоенные диаграммы (19) и (20) дают декартов квадрат

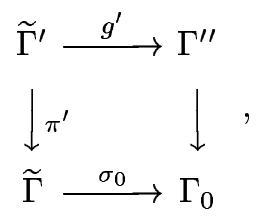

в котором $\Gamma^{\prime \prime}$ есть расслоенное произведение $\Gamma_{0} \times \widetilde{H}^{\prime} H^{\prime}$ с естественным вложением $\Gamma^{\prime \prime} \hookrightarrow$ $S \times H^{\prime}$, так что $\widetilde{\Gamma}^{\prime}=\Gamma^{\prime \prime} \times{ }_{S} \widetilde{S}$, а проекция $g^{\prime}$ индуцирована нижней проекцией $\sigma \times \operatorname{id}_{H^{\prime}}$ 
в диаграмме (21); тем самым, ввиду неприводимости $\widetilde{\Gamma}^{\prime}$ многообразие $\Gamma^{\prime \prime}$ также неприводимо и ввиду $(22)$ совпадает с $\Gamma^{\prime}$ (соответственно $g^{\prime}=g$ ). Итак, имеем диаграмму расслоенных произведений:

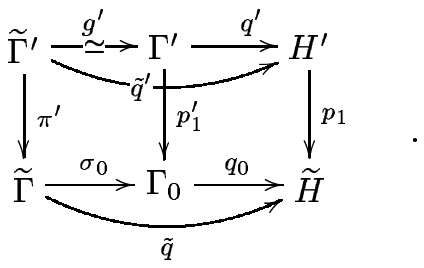

Так как $q^{\prime}$ - конечный плоский морфизм степени $d$, то из этой диаграммы следует, что

$$
E^{\prime}:=q_{*}^{\prime} \mathscr{O}_{\Gamma^{\prime}}=q_{*}^{\prime} p_{1}^{\prime *} \mathscr{O}_{\Gamma_{0}}=p_{1}^{*} q_{0 *} \mathscr{O}_{\Gamma_{0}}=p_{1}^{*} \operatorname{pr}_{2 *} \mathscr{O}_{\Gamma_{0}}
$$

- локально свободньй $\mathscr{O}_{H^{\prime}}$-пучок степени $d$. Заметим также, что нижние треугольники в диаграммах (10) и (24) совпадают. Поэтому, применяя к точной тройке (8) функтор $p_{1}^{*} \operatorname{pr}_{2 *}$ и учитывая (14) и $(25)$, получаем точную тройку:

$$
0 \rightarrow E^{\prime} \rightarrow p_{1}^{*} E \rightarrow p_{1}^{*} F \rightarrow 0
$$

Тем самым, поскольку $p_{1}^{*} E$ и $E^{\prime}$ - локально свободные $\mathscr{O}_{H^{\prime}}$-пучки степени $d$, а $H^{\prime}$ - неприводимое многообразие, то пучок $p_{1}^{*} F$ является $\mathscr{O}_{H^{\prime}}$-пучком кручения гомологической размерности 1 , а значит, по лемме 1.2 пучок идеалов Фиттинга

$$
\operatorname{Fitt}^{0}\left(p_{1}^{*} F\right)=p_{1}^{-1} \operatorname{Fitt}^{0}(F) \cdot \mathscr{O}_{H^{\prime}}=p_{1}^{-1} \mathbf{J} \cdot \mathscr{O}_{H^{\prime}}
$$

обратим. Поэтому по универсальному свойству раздутий (см., например, [2, гл. 2, 7.14]) существует единственный морфизм $\phi: H^{\prime} \rightarrow \widehat{H}$ такой, что $p_{1}=\delta \circ \phi$. Из диаграммы (18) теперь следует, что проекция $p_{2}:=\gamma \circ \phi$ удовлетворяет требуемому условию $p_{2}=f \circ p_{1}$. Итак, доказана

ТЕОРема 1.3. Пусть $\Gamma_{f}-$ замыкание в $\widetilde{H} \times H$ графика бирационального изоморфизма схем Гильберта $f: \widetilde{H} \rightarrow H$, индуиируемого раздутием $\sigma: \widetilde{S} \rightarrow S$ точки $x_{0}$ в S. Тогда $\Gamma_{f}$ изоморфно раздутию схемь Гильберта $\widetilde{H}$ в подсхеме $\left(R, \mathscr{O}_{R}\right)$, где $R:=\left\{z \in \widetilde{H} \mid \operatorname{length}\left(Z \cap l_{0}\right) \geqslant 2\right\}-$ иикл иниидениии, а $\mathscr{O}_{R}=\mathscr{O}_{\widetilde{H}} / \mathbf{J}$, где $\mathbf{J}$ есть пучок нулевых идеалов Фиттинга $\mathbf{J}=\operatorname{Fitt}^{0}\left(\operatorname{pr}_{2 *} R^{1} \bar{\sigma}_{*} \mathscr{J}_{\widetilde{\Gamma}, \widetilde{S} \times \tilde{H}}\right)$.

В случае $d=2$ из теоремы 1.3 и замечания 1.1 непосредственно вытекает

СледСТВИЕ 1.4. При $d=2$ многообразие $\Gamma_{f}$ является гладким $и \operatorname{pr}_{\widetilde{H}}: \Gamma_{f} \rightarrow \widetilde{H}-$ раздутие $\widetilde{H}$ в гладкой подсхеме $R=\mathrm{Hilb}^{2} l_{0}$, изоморфной $\mathbf{P}^{2}$. 
2. Случай длины $d=2$. В этом пункте мы рассматриваем случай $d=2$. Согласно следствию 1.4 в этом случае $\Gamma_{f}$ - гладкое многообразие. Более того, оказывается, что морфизм $\operatorname{pr}_{H}: \Gamma_{f} \rightarrow H$ разлагается в композицию двух раздутий с гладкими центрами. Цель этого пункта - получить точное описание этих разложений. Для этого в схеме Гильберта $H=H_{2}$ рассмотрим замкнутое подмножество $Q=\left\{z \in H \mid Z \ni x_{0}\right\}$ (цикл инциденции). Легко видеть, что $Q$ как приведенная подсхема в $H$ изоморфна $\widetilde{S}$, и при этом изоморфизме

$$
Q \stackrel{\sim}{\rightarrow} \widetilde{S}
$$

имеем

$$
\left\{z \in Q \mid \operatorname{Supp} Z=x_{0}\right\} \stackrel{\sim}{\rightarrow} l_{0} .
$$

Пусть $\Gamma=\{(x, z) \in S \times H \mid x \in Z\}$ - универсальньй цикл (см. [1]) с проекциями $S \stackrel{p}{\leftarrow} \Gamma \stackrel{q}{\rightarrow} H$, рассматриваемьй как приведенная неприводимая подсхема в $S \times H$. (Аналогично имеем универсальньй цикл $\widetilde{\Gamma} \subset \widetilde{S} \times \widetilde{H}$ с проекциями $\widetilde{S} \stackrel{\widetilde{p}}{\leftarrow} \stackrel{\widetilde{q}}{\rightarrow} \widetilde{H}$.) Рассмотрим раздутие $\sigma_{Q}: H^{\prime} \rightarrow H$ схемы $H$ с центром в $Q$ как гладкой подсхеме, и пусть $D_{Q}=\sigma^{-1}(Q)$ - исключительньй дивизор раздутия $\sigma$. Используя отождествления (26) и $(27)$, имеем линейчатую поверхность $F=\sigma_{Q}^{-1}\left(l_{0}\right)=\mathbf{P}\left(N_{Q / H} \mid l_{0}\right)$ с проекцией

$$
\pi_{F}=\sigma_{Q} \mid F: F \rightarrow l_{0} \simeq \mathbf{P}^{1}
$$

и коммутативную диаграмму

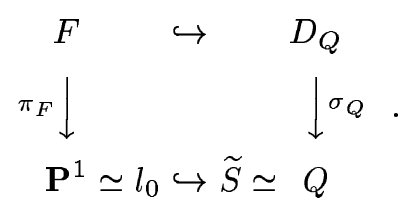

ЛЕмма 2.1. Имеем

$$
N_{Q / H} \mid l_{0} \simeq \mathscr{O}_{\mathbf{P}^{1}}(2) \oplus \mathscr{O}_{\mathbf{p}^{1}}(-1)
$$

так что поверхность $F$ есть поверхность типа $\mathbb{F}_{3}$. При этом $F$ канонически изоморфна многообразию полных пунктуальных флагов подсхем длины 1,2 и 3 на $S$ с носителем в точке $x_{0}$.

ДокАЗАТЕЛЬСтво. Действительно, для произвольной точки $z \in l_{0} \subset Q$ соответствующая схема $Z \subset S$ включается в точную тройку ${ }^{2}$

$$
0 \rightarrow \mathscr{I}_{x_{0}} \rightarrow \mathscr{O}_{S} \rightarrow k_{x_{0}} \rightarrow 0
$$

Применяя к этой тройке функтор $\operatorname{Hom}\left(\cdot, \mathscr{O}_{Z}\right)$, имеем

$$
0 \rightarrow \operatorname{Hom}\left(k_{x_{0}}, \mathscr{O}_{Z}\right) \rightarrow \operatorname{Hom}\left(\mathscr{O}_{S}, \mathscr{O}_{Z}\right) \rightarrow \operatorname{Hom}\left(\mathscr{I}_{x_{0}}, \mathscr{O}_{Z}\right) \rightarrow \operatorname{Ext}^{1}\left(k_{x_{0}}, \mathscr{O}_{Z}\right) \rightarrow 0
$$

\footnotetext{
${ }^{2}$ Здесь и ниже через $k_{x}$ будем обозначать поле вычетов точки $x$ на данной схеме $X$.
} 
Легко видеть с учетом двойственности Серра на $S$ и равенства

$$
\sum_{i=0}^{2}(-1)^{i} \operatorname{dim} \operatorname{Ext}^{i}\left(k_{x_{0}}, \mathscr{O}_{Z}\right)=0
$$

что

$$
\operatorname{Hom}\left(k_{x_{0}}, \mathscr{O}_{Z}\right)=k, \quad \operatorname{Ext}^{2}\left(k_{x_{0}}, \mathscr{O}_{Z}\right)=\operatorname{Hom}\left(\mathscr{O}_{Z}, k_{x_{0}}\right)^{\vee}=k, \quad \operatorname{Ext}^{1}\left(k_{x_{0}}, \mathscr{O}_{Z}\right)=k^{2} .
$$

Далее, беря произвольную точку $z \in Q$, где $\operatorname{Supp} Z=x_{0} \cup x_{1}$, и применяя функтор $\operatorname{Hom}\left(\cdot, \mathscr{O}_{Z}\right)$ к тройке

$$
0 \rightarrow \mathscr{I}_{Z} \rightarrow \mathscr{I}_{x_{0}} \rightarrow k_{x_{1}} \rightarrow 0
$$

получаем точную последовательность

$$
0 \rightarrow \operatorname{Hom}\left(k_{x_{1}}, \mathscr{O}_{Z}\right) \rightarrow \operatorname{Hom}\left(\mathscr{I}_{x_{0}}, \mathscr{O}_{Z}\right) \rightarrow \operatorname{Hom}\left(\mathscr{I}_{Z}, \mathscr{O}_{Z}\right) \rightarrow \operatorname{Ext}^{1}\left(k_{x_{1}}, \mathscr{O}_{Z}\right) \rightarrow \cdots
$$

Эта последовательность и естественные изоморфизмы

$$
T_{z} Q \simeq \operatorname{Hom}\left(\mathscr{I}_{x_{0}}, \mathscr{O}_{Z}\right) / \operatorname{Hom}\left(k_{x_{1}}, \mathscr{O}_{Z}\right) \simeq k^{2}, \quad T_{z} H \simeq \operatorname{Hom}\left(\mathscr{I}_{Z}, \mathscr{O}_{Z}\right) \simeq k^{4}
$$

вместе с равенством $\operatorname{Ext}^{1}\left(k_{x_{1}}, \mathscr{O}_{Z}\right)=k^{2}$, получаемым из последнего равенства (31) заменой $x_{0}$ на $x_{1}$, дают изоморфизм

$$
\left.N_{Q / H}\right|_{z} \simeq \operatorname{Ext}^{1}\left(k_{x_{1}}, \mathscr{O}_{Z}\right) .
$$

Для получения релятивизированного варианта равенства (34) рассмотрим семейство двоеточий $\Gamma_{l_{0}}=\Gamma \times{ }_{H} l_{0}$ с носителем $x_{0}$ как подсхему в $S \times l_{0} \simeq S \times \mathbf{P}^{1}$ и положим $\mathscr{E}:=\mathcal{E} x t_{\mathrm{pr}_{2}}^{1}\left(k_{x_{0}} \otimes \mathscr{O}_{\mathbf{p}^{1}}, \mathscr{O}_{\Gamma_{l_{0}}}\right)$, где $\mathrm{pr}_{2}: S \times \mathbf{P}^{1} \rightarrow \mathbf{P}^{1}$-проекция. Замена базы для функтоpa $\mathcal{H}$ pr $_{\mathrm{pr}_{2}}\left(\cdot, \mathscr{O}_{\Gamma_{l_{0}}}\right)$ ввиду равенств (31) дает канонический изоморфизм $\left.\left.N_{Q / H}\right|_{z} \simeq \mathscr{E}\right|_{z}$, $z \in l_{0}$. Более того, тройка $(29)$ релятивизируется в $\mathscr{O}_{S \times \mathbf{P}^{1-т р о и ̆ к у ~}}$

$$
0 \rightarrow \mathscr{I}_{x_{0} \times \mathbf{P}^{1}} \rightarrow \mathscr{O}_{S \times \mathbf{P}^{1}} \rightarrow k_{x_{0}} \otimes \mathscr{O}_{\mathbf{p}^{1}} \rightarrow 0,
$$

и применение к последней функтора $\mathcal{H o m}_{\mathrm{pr}_{2}}\left(\cdot, \mathscr{O}_{\Gamma_{l_{0}}}\right)$ дает длинную точную последова-

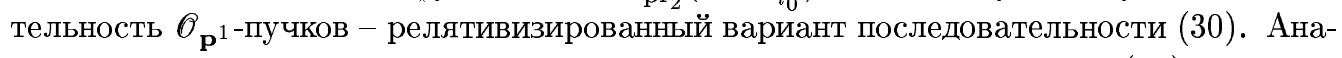
логично получаем и релятивизированный вариант последовательности (33), и предыдущие рассуждения дают канонический изоморфизм расслоений:

$$
N_{Q / H} \mid l_{0} \simeq \mathscr{E} .
$$

Теперь совпадение поверхности $F=P\left(N_{Q / H} \mid l_{0}\right)$ с многообразием полных пунктуальных флагов подсхем длины 1,2 и 3 на $S$ с носителем в точке $x_{0}$ следует из основного результата работы [5] (см. также [6]). В той же работе [5] доказано равенство $\mathscr{E}=\mathscr{O}_{\mathbf{p}^{1}}(2) \oplus \mathscr{O}_{\mathbf{p}^{1}}(-1)$ и, тем самьм, совпадение $F$ с $\mathbb{F}_{3}$. 
ЗАмЕчАниЕ 2.2. Для произвольной точки $z \in Q \backslash l_{0}$, как легко видеть, определен канонический изоморфизм $\left.N_{Q / H}\right|_{z} \simeq T_{x_{0}} S$, так что слой $\sigma_{Q}^{-1}(z)$ изоморфен $l_{0}$. Таким образом, точки из $D_{Q} \backslash F$ параметризуют подсхемы длины 2 в $\widetilde{S}$, состоящие из двух различных точек, из которых в точности одна лежит на $l_{0}$.

Рассмотрим диаграмму расслоенных произведений

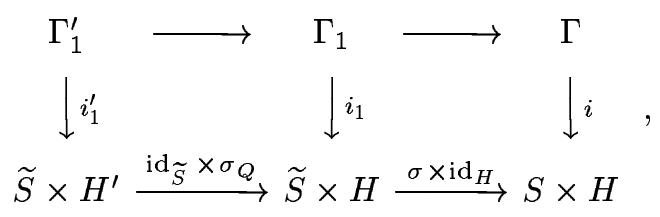

где $i$ - естественное вложение, так что $i_{1}$ и $i_{1}^{\prime}$ - также вложения. Пусть $D_{1}^{\prime}=\Gamma_{1}^{\prime} \times{ }_{H^{\prime}} D_{Q}$, $\Gamma^{\prime}=\overline{\Gamma_{1}^{\prime} \backslash D_{1}^{\prime}}$ (замькание берется в $\left.\widetilde{S} \times H^{\prime}\right)$ и $\gamma^{\prime}:=q^{\prime} \mid \Gamma^{\prime}: \Gamma^{\prime} \rightarrow H^{\prime}$ - проекция. Далее, согласно лемме 2.1 на поверхности $F$ в $H^{\prime}$ имеем исключительную прямую $m_{0}$, определяемую условием $\left(m_{0}^{2}\right)_{F}=-3$.

Лемма 2.3. Морфизм $\gamma^{\prime}: \Gamma^{\prime} \rightarrow H^{\prime}$ конечен степени 2 над $H^{\prime} \backslash m_{0}$ и имеет слои $\mathbf{P}^{1}$ над точками прямой $m_{0}$.

ДокАЗАТЕЛЬСтво. Проведем доказательство локальньми вычислениями в подходящей открытой окрестности на $S \times H$. Выберем на $S$ локальные параметры $u, v$ в точке $x_{0}$, т.е. функции, регулярные в некоторой окрестности $U \subset S$ точки $x_{0}$ такие, что отображение $\phi: U \rightarrow \mathbf{A}^{2}$, при котором $x \mapsto(u(x), v(x))$, имеет невырожденный дифференциал $\left.d \phi\right|_{x_{0}}$ в точке $x_{0}$. В силу открытости последнего условия можно считать, что $\left.d \phi\right|_{x}$ невырожден в каждой точке $x \in U$, т.е. вьполнено условие:

$(*)$ для произвольной точки $x \in U$ функции $u-u(x), v-v(x)$ являются локальными параметрами в точке $x$.

Уменьшив $U$, если нужно, можем считать также, что вьполнено условие:

$(* *) \phi^{-1}(0,0)=x_{0}$.

Далее, для произвольной точки $(a, b) \in \mathbf{A}^{2}$ рассмотрим в $U$ кривую

$$
C_{a, b}=\{x \in U \mid v(x)-a u(x)-b=0\}
$$

Из условия $(*)$ следует, что если $C_{a, b} \neq \varnothing$, то $C_{a, b}-$ гладкая кривая в $U$. В частности, $\varnothing \neq C_{0,0}$ - гладкая кривая в $U$, содержащая точку $x_{0}$. Тем самьг, однозначно определена точка $z_{0} \in H$ такая, что

$$
Z_{0} \subset C_{0,0}, \quad \operatorname{Supp} Z_{0}=x_{0}
$$

Пусть $Z \subset U$ - произвольная подсхема длины 2 , Supp $Z=x_{1} \cup x_{2}$. Определим на $H$ рациональную функцию $a: H \rightarrow k$ следующим образом:

(i) если точки $x_{1}$ и $x_{2}$ различны, то полагаем

$$
a(z)=\frac{\left(v\left(x_{1}\right)-v\left(x_{2}\right)\right)}{\left(u\left(x_{1}\right)-u\left(x_{2}\right)\right)}
$$

(ii) если $x_{1}=x_{2}$, то ввиду условия $(*)$ через точку $\phi\left(x_{1}\right)$ проходит единственная кривая $C_{a_{1}, b_{1}}$ такая, что $d \phi\left(T_{x_{1}} Z\right)=T_{\phi\left(x_{1}\right)} C_{a_{1}, b_{1}}$ (и в этом случае $\left.b_{1}=v\left(x_{1}\right)-a_{1} u\left(x_{1}\right)\right)$; 
в этом случае полагаем $a(z)=a_{1}$. Легко проверяется корректность этого определения. Рассмотрим в $H$ открытое подмножество $V^{\prime}$, на котором функция $a$ регулярна, и в нем открытое подмножество $V=\left\{z \in V^{\prime} \mid Z \subset U\right\}$. Определим на $V$ наряду с $а$ еще три регулярные функции $b, c, d$ следуюшим образом:

$$
\begin{gathered}
b(z)=v\left(x_{1}\right)-a(z) u\left(x_{1}\right)=v\left(x_{2}\right)-a(z) u\left(x_{2}\right) \\
c(z)=u\left(x_{1}\right)+u\left(x_{2}\right), \quad d(z)=-u\left(x_{1}\right) u\left(x_{2}\right)
\end{gathered}
$$

Из (37), (38), (39) следует, что $z_{0} \in V$, причем в силу $(*)$ и (**) система уравнений $a=b=c=d=0$ имеет в $V$ единственное решение $z_{0}$. Поэтому, уменьшив $V$ подходящим образом, мы можем считать, что

$(* * *) a-a(z), b-b(z), c-c(z), d-d(z)$ - локальные параметры в каждой точке $z \in V$.

Открытое множество $U \times V$ - подходящая окрестность точки $\left(x_{0}, z_{0}\right) \in S \times H$, в которой мы будем проводить дальнейшие вычисления. Из (38) и (39) непосредственно получаем уравнения универсального цикла $\Gamma \cap(U \times V)$ в $U \times V$ :

$$
v(x)-a u(x)-b=u^{2}(x)+c u(x)+d=0
$$

соответственно, уравнения поверхности $Q \cap V$ и прямой $l_{0} \cap V$ в $V$ имеют вид

$$
Q \cap V: \quad b=d=0, \quad l_{0} \cap V: \quad b=c=d=0 .
$$

Перейдем к локальньм формулам раздутий $\sigma: \widetilde{S} \rightarrow S$ и $\sigma_{Q}: H^{\prime} \rightarrow H$. Возьмем произвольную точку $x^{\prime} \in l_{0}$. Как известно (см., например, [7, дополнение В.6.10]), в подходящей окрестности $U^{\prime} \subset \sigma^{-1}(U)$ точки $x^{\prime}$ можно выбрать локальные параметры $u^{\prime}, v^{\prime}$ так, что уравнения раздутия $\sigma: U^{\prime} \rightarrow U$ имеют вид

$$
u=u^{\prime}, \quad v=u^{\prime} v^{\prime}
$$

Соответственно, в подходящей окрестности $V^{\prime} \subset \sigma_{Q}^{-1}(V)$ произвольной точки $z^{\prime} \in$ $\sigma_{Q}^{-1}\left(z_{0}\right)$ выберем локальные параметры $a^{\prime}, b^{\prime}, c^{\prime}, d^{\prime}$, в которых уравнения раздутия $\sigma_{Q}: V^{\prime} \rightarrow V$ имеют вид

$$
a=a^{\prime}, \quad b=b^{\prime}, \quad c=c^{\prime}, \quad d=b^{\prime} d^{\prime}
$$

соответственно,

$$
D_{Q} \cap V^{\prime}: \quad b^{\prime}=0, \quad F \cap V^{\prime}: \quad b^{\prime}=c^{\prime}=0 .
$$

Кроме того, элементарное вьгисление дает

$$
m_{0} \cap V^{\prime}: \quad b^{\prime}=c^{\prime}=d^{\prime}=0 .
$$

Из (40), (42) и (43) находим

$$
\Gamma_{1}^{\prime} \cap\left(U^{\prime} \times V^{\prime}\right): \quad u^{\prime} v^{\prime}-a^{\prime} u^{\prime}-b^{\prime}=u^{\prime 2}+c^{\prime} u^{\prime}+b^{\prime} d^{\prime}=0 .
$$


Обозначим через $q_{1}^{\prime}$ естественную проекцию $\Gamma_{1}^{\prime} \rightarrow H^{\prime}$. Из (44) и первого уравнения (46) находим

$$
q_{1}^{\prime-1}\left(D_{Q}\right) \cap\left(U^{\prime} \times V^{\prime}\right)=\left\{u^{\prime}=0\right\}
$$

так что уравнения пересечения $\Gamma_{1}^{\prime} \cap\left(U^{\prime} \times V^{\prime}\right)$ в $U^{\prime} \times V^{\prime}$ получаются подстановкой выражения для $b^{\prime}$ из первого уравнения (46) во второе и делением последнего на $u^{\prime}$ :

$$
\Gamma_{1}^{\prime} \cap\left(U^{\prime} \times V^{\prime}\right): \quad b^{\prime}-u^{\prime}\left(v^{\prime}-a^{\prime}\right)=u^{\prime}+c^{\prime}+d^{\prime}\left(v^{\prime}-a^{\prime}\right)=0 .
$$

Пусть $\mathrm{pr}: U^{\prime} \times V^{\prime} \stackrel{\operatorname{pr}_{1}}{\rightarrow} U^{\prime} \stackrel{\phi}{\rightarrow} \mathbf{A}^{2}-$ проекция. Из (47) для произвольной точки $\left(a^{\prime}, 0, c^{\prime}, d^{\prime}\right) \in$ $D_{Q} \cap V^{\prime}$ находим

$$
\gamma^{\prime-1}\left(a^{\prime}, 0, c^{\prime}, d^{\prime}\right)=x_{1}^{\prime} \cup x_{2}^{\prime}, \quad \operatorname{pr}\left(x_{1}^{\prime}\right)=\left(-c^{\prime}, a^{\prime}\right), \quad \operatorname{pr}\left(x_{2}^{\prime}\right)=\left(0, a^{\prime}-c^{\prime} / d^{\prime}\right) .
$$

Соответственно, $(45),(47)$ и (48) показывают, что для произвольной точки $\left(a^{\prime}, 0,0,0\right) \in$ $m_{0} \cap V^{\prime}$ находим

$$
\operatorname{pr}_{1}\left(\gamma^{\prime-1}\left(a^{\prime}, 0,0,0\right) \cap\left(U^{\prime} \times V^{\prime}\right)\right)=\left\{u^{\prime}=0\right\}=l_{0} \cap U^{\prime} .
$$

Аналогичные вычисления проводим в других локальных картах. Лемма доказана.

СЛЕДСТВИЕ 2.4. Имеет место естественный изоморфизм схем:

$$
F \backslash m_{0} \simeq\left\{z \in \widetilde{H} \mid \operatorname{Supp} Z=\{p t\} \in l_{0}, Z \not \subset l_{0}\right\} .
$$

ДоКАЗАТЕЛЬСтво. Достаточно провести вычисление в любой из карт. Согласно $(44)$ и (45) всякая точка $z \in\left(F \backslash m_{0}\right) \cap V^{\prime}$ имеет вид $z=\left(a^{\prime}, 0,0, d^{\prime}\right), d^{\prime} \neq 0$; отсюда и из $(41),(43),(48)$ и (46) следует, что схема $Z=\gamma^{\prime-1}(z)$ имеет носитель в точке $x_{1}^{\prime} \in l_{0}$, где $\operatorname{pr}\left(x_{1}^{\prime}\right)=\left(0, a^{\prime}\right)$, т.е. $a^{\prime}$ есть аффинная координата на $l_{0}$; соответственно, $d^{\prime}$ есть аффинная координата на проективной прямой $P\left(T_{x_{1}^{\prime}} \widetilde{S}\right)$ такая, что точка $P\left(T_{x_{1}^{\prime}} l_{0}\right)$ имеет координату $d^{\prime}=0$, а точка $P\left(T_{x_{1}^{\prime}} Z\right)$ - координату $-d^{\prime}$. Поэтому условие $d^{\prime} \neq 0$ означает, что $F \backslash m_{0}$ параметризует неприведенњые схемы длины 2 в $\widetilde{S}$ с носителем на $l_{0}$, не лежащие на $l_{0}$.

Рассмотрим многообразие $R=\operatorname{Hilb}^{2} l_{0} \simeq S^{2} l_{0}$ (см. замечание 1.1 ) и его естественное вложение $R \hookrightarrow \widetilde{H}$. Так как $l_{0} \simeq P\left(T_{x_{0}} S\right) \simeq \mathbf{P}^{1}$, имеем канонический изоморфизм $R \simeq \mathbf{P}^{2}$. (Более того, несложное вычисление показывает, что $N_{R / \tilde{H}} \simeq \mathscr{O}_{\mathbf{P}^{2}}(-1)^{\oplus 2}$.) Далее, из следствия 2.4 и замечания 2.2 вытекает естественньй изоморфизм многообразий

$$
\tilde{H} \backslash R \stackrel{\sim}{\rightarrow} H^{\prime} \backslash m_{0}
$$

Рассмотрим раздутие $\sigma_{m_{0}}: H^{\prime \prime} \rightarrow H^{\prime}$ с центром в $m_{0}$, и пусть $D_{m_{0}}=\sigma_{m_{0}}^{-1}\left(m_{0}\right)-$ исключительньй дивизор на $H^{\prime \prime}, \Gamma_{2}=H^{\prime \prime} \times_{H^{\prime}} \Gamma^{\prime}$ и $D_{2}=\Gamma_{2} \times_{H^{\prime \prime}} D_{m_{0}}$ - дивизор на $\Gamma_{2}$. Считая $\Gamma_{2}$ естественно вложенным в $\widetilde{S} \times H^{\prime \prime}$, рассмотрим многообразие $\Gamma^{\prime \prime}=\overline{\Gamma_{2} \backslash D_{2}}$ (замыкание берется в $\widetilde{S} \times H^{\prime \prime}$ ), и пусть $\gamma^{\prime \prime}: \Gamma^{\prime \prime} \rightarrow H^{\prime \prime}$ - естественная проекция.

Лемма 2.5. Отображсене $\gamma^{\prime \prime}: \Gamma^{\prime \prime} \rightarrow H^{\prime \prime}$ - конечный плоский морфизм степени 2 . 
ДокАЗАТЕльство. Воспользуемся обозначениями из доказательства леммы 2.3. Пусть $V^{\prime \prime} \subset \sigma_{m_{0}}^{-1}\left(V^{\prime}\right)$ - локальная карта в $H^{\prime \prime}$ с локальньми параметрами $a^{\prime \prime}, b^{\prime \prime}, c^{\prime \prime}$, $d^{\prime \prime}$, в которых раздутие $\sigma_{m_{0}}$ задается уравнениями

$$
a^{\prime}=a^{\prime \prime}, \quad b^{\prime}=b^{\prime \prime}, \quad c^{\prime}=b^{\prime \prime} c^{\prime \prime}, \quad d^{\prime}=b^{\prime \prime} d^{\prime \prime} .
$$

Из (52) и (47) получаем уравнения $\Gamma_{2} \cap\left(U^{\prime} \times V^{\prime \prime}\right)$ в $U^{\prime} \times V^{\prime \prime}$ :

$$
\Gamma_{2} \cap\left(U^{\prime} \times V^{\prime \prime}\right): \quad b^{\prime \prime}-u^{\prime}\left(v^{\prime}-a^{\prime \prime}\right)=0, \quad u^{\prime}+b^{\prime \prime} c^{\prime \prime}+b^{\prime \prime} d^{\prime \prime}\left(v^{\prime}-a^{\prime \prime}\right)=0 .
$$

Отсюда по аналогии с (47) находим уравнения $\Gamma^{\prime \prime} \cap\left(U^{\prime} \times V^{\prime \prime}\right)$ в $U^{\prime} \times V^{\prime \prime}$ :

$$
\Gamma^{\prime \prime} \cap\left(U^{\prime} \times V^{\prime \prime}\right): \quad b^{\prime \prime}-u^{\prime}\left(v^{\prime}-a^{\prime \prime}\right)=0, \quad 1+c^{\prime \prime}\left(v^{\prime}-a^{\prime \prime}\right)+d^{\prime \prime}\left(v^{\prime}-a^{\prime \prime}\right)=0 .
$$

Отсюда сразу следует, что слой проекции $\gamma^{\prime \prime}: \Gamma^{\prime \prime} \cap\left(U^{\prime} \times V^{\prime \prime}\right)$ над произвольной точкой дивизора $D_{m_{0}} \cap V^{\prime \prime}=\left\{b^{\prime \prime}=0\right\}$ состоит из двух точек (не обязательно различных). Таким образом, $\gamma^{\prime \prime}-$ конечный морфизм степени 2. Так как каждьй слой морфизма $\gamma^{\prime \prime}$ имеет постоянную схемную длину 2 , то, значит, $\gamma^{\prime \prime}$ - плоский морфизм.

Из леммы 2.5 и универсальности схемы Гильберта $\widetilde{H}$ теперь немедленно следует, что определен морфизм $\delta: H^{\prime \prime} \rightarrow \widetilde{H}$ такой, что $\Gamma^{\prime \prime}=\left(\operatorname{id}_{\widetilde{S}} \times \delta\right)^{-1}(\widetilde{\Gamma})$, где $\widetilde{\Gamma} \subset \widetilde{S} \times \widetilde{H}-$ универсальный цикл над $\widetilde{H}$. При этом из (51) следует, что $\delta: H^{\prime \prime} \backslash D_{m_{0}} \rightarrow \widetilde{H} \backslash R$-изоморфизм. Тем самьм, ввиду гладкости и неприводимости многообразий $R$ и $D_{m_{0}}$ имеем

$$
D_{m_{0}}=\delta^{-1}(R) \text {. }
$$

Пусть $\operatorname{pr}_{\widetilde{H}}: \widehat{H} \rightarrow \widetilde{H}$ - раздутие с центром в $R$ как гладкой подсхеме (следствие 1.4$)$ и $D_{R}:=\operatorname{pr}_{\tilde{H}}^{-1}(R)$. Так как $D_{m_{0}}$ - дивизор на гладком многообразии $H^{\prime \prime}$, то из универсального свойства раздутий [2, гл. $2,7.14]$ следует, что определен морфизм (регулярньй бирациональньй) $\psi: H^{\prime \prime} \rightarrow \Gamma_{f}$ такой, что $\delta=\operatorname{pr}_{\tilde{H}} \circ f$, причем

(i) $\psi^{-1}\left(D_{R}\right)=D_{m_{0}}$

(ii) $\psi: H^{\prime \prime} \backslash D_{m_{0}} \rightarrow \Gamma_{f} \backslash D_{R}-$ изоморфизм.

Нетрудно видеть, что $\psi$ - изоморфизм. В самом деле, в противном случае ввиду гладкости $\Gamma_{f}$ и известного свойства бирациональных морфизмов (см., например, [8, гл. 2, $\S 4$, теорема 2]) существует непустое замкнутое неприводимое подмножество $Z$ коразмерности $\geqslant 2$ в $\Gamma_{f}$ такое, что $D=\psi^{-1}(Z)$ - дивизор в $H^{\prime \prime}$; при этом в силу (ii) $Z \subset D_{R}$, а значит, $D \subset \psi^{-1}\left(D_{R}\right)$; тем самым, $\psi^{-1}\left(D_{R}\right)$ содержит кроме $D$ еще по крайней мере одну компоненту $\overline{\psi^{-1}\left(D_{R} \backslash Z\right)}$ (замыкание в $H^{\prime \prime}$ ) вопреки условию $(\mathrm{i})$, поскольку $D_{m_{0}}$ неприводим. Итак, $\psi$ - изоморфизм, т.е. можно считать, что $H^{\prime \prime}=\Gamma_{f}$ и $\delta$ совпадает с $\operatorname{pr}_{\tilde{H}}$, и мы получаем следующий основной результат этого пункта.

Теорема 2.6. Пусть $\sigma: \widetilde{S} \rightarrow S$-раздутие с иентром в точке $x_{0} \in S$ и $l_{0}=$ $\sigma^{-1}\left(x_{0}\right)$. Пусть $d=2, \sigma_{Q}: H^{\prime} \rightarrow H-$ раздутие схемь Гильберта $H=$ Hilb $^{2} S$ с иентром в гладкой поверхности $Q=\left\{z \in H \mid Z \ni x_{0}\right\}, l_{0} \hookrightarrow \widetilde{S} \simeq Q-$ вложсение исключительной прямой в $Q, F=\sigma_{Q}^{-1}\left(l_{0}\right)$ - линейчатая поверхность типа $\mathbb{F}_{3}$ и то $\subset F$ - исключительная прямая на $F$. Тогда определен морфизм $\sigma_{m_{0}}: \Gamma_{f} \rightarrow H^{\prime}$, являющийся раздутием с гладким иентром $m_{0}$, такой, что проекиия $\mathrm{pr}_{H}: \Gamma_{f} \rightarrow H$ разлагается в композицию раздутий $\sigma_{m_{0}}$ и $\sigma_{Q}$ с гладкими иентрами:

$$
\operatorname{pr}_{H}=\sigma_{Q} \circ \sigma_{m_{0}}
$$


3. Общий случай длины $d \geqslant 3$. В общем случае $d \geqslant 3$ цикл инциденции $R$ в $\widetilde{H}$, рассматриваемьй как неприводимое многообразиес приведенной структурой $R=R_{\text {red }}$, уже не является неособым и нетрудно видеть, что его особенности имеют вид:

$$
Y:=\operatorname{Sing} R_{\text {red }}=\left\{z \in \widetilde{H} \mid \operatorname{length}\left(Z \cap l_{0}\right) \geqslant 3\right\}
$$

Тем самым, в общей точке $z \in Y$ многообразие $R_{\text {red }}$ локально аналитически изоморфно $Y \times T$, где $T$ - объединение трех координатных осей в $\mathbf{A}^{3}$ (с приведенной структурой). Поэтому, как показывает простое вычисление, результат раздутия $\widetilde{H}$ с центром в $R_{\text {red }}$ является особым многообразием. Далее, рассмотрим в $\mathbf{A}^{3}$ координатные плоскости $V_{1}=\{x=0\}, V_{2}=\{y=0\}, V_{3}=\{z=0\}$ и их объединение $V=\{x y z=0\}$, и пусть

$$
g=\left(\operatorname{res}_{V_{1}}, \operatorname{res}_{V_{2}}, \operatorname{res}_{V_{3}}\right): \mathscr{O}_{V} \rightarrow \mathscr{O}_{V_{1}} \oplus \mathscr{O}_{V_{2}} \oplus \mathscr{O}_{V_{3}}
$$

- естественньй морфизм. Нетрудно видеть, что в окрестности общей точки $z \in Y$ пучок $F$ из (28) локально аналитически изоморфен пучку $\mathscr{O}_{Y} \otimes \mathscr{F}_{0}$, где $\mathscr{F}_{0}:=\operatorname{Coker}(g)$. С другой стороны, из определения $\mathscr{F}_{0}$ легко получаем, что (при подходящей нормировке компонент $\operatorname{res}_{V_{i}}$ морфизма $g$ ) пучок $\mathscr{F}_{0}$ изоморфен коядру морфизма $\mathscr{O}_{\mathbf{A}^{3}}^{\oplus 3} \rightarrow \mathscr{O}_{\mathbf{A}^{3}}^{\oplus 2}$, задаваемого $(2 \times 3)$-матрицей

$$
M=\left(m_{i j}\right), \quad \text { где } m_{11}=x, m_{12}=y, m_{13}=-2 z, m_{21}=-2 x, m_{22}=y, m_{23}=z .
$$

Тем самым, элементарньм вычислением получаем, что пучок идеалов Фиттинга $\operatorname{Fitt}^{0}\left(\mathscr{F}_{0}\right)$ локально изоморфен пучку идеалов объединения координатных осей в $\mathbf{A}^{3}$ (т.е. имеет образующие $x y, x z, y z$ ); ввиду вышесказанного, это равносильно тому, что пучок идеалов Фиттинга $\mathbf{J}=$ Fitt $^{0} F$ локально аналитически в окрестности общей точки $z \in Y$ изоморфен пучку идеалов приведенной схемы $R_{\text {red }}$. Тем самым, мы получаем следующий результат.

ТЕОРема 3.1. При $d \geqslant 3 \Gamma_{f}$ является особим многообразием.

\section{СПИСОК ЦИТИРОВАННОЙ ЛИТЕРАТУРЫ}

[1] Fogarty J. Algebraic families on an algebraic surface, II: The Picard scheme of the punctual Hilbert scheme // Amer. J. Math. 1973. V. 95. P. 660-687.

[2] Хартсхорн Р. Алгебраическая геометрия. М.: Мир, 1984.

[3] Тихомиров А. С. Многообразие полных пар нульмерных подсхем алгебраической поверхности // Изв. РАН. Сер. матем. 1997. Т. 61. №6. С. 153-180.

[4] Мамфорд Д. Лекции о кривых на алгебраической поверхности. М.: Мир, 1968.

[5] Тихомиров С.А. Пунктуальные схемы Гильберта малой длины в размерностях 2 и $3 / /$ Матем. заметки. 2000. Т. 67. №3. С. 414-432.

[6] Тихомиров А. С. Гладкая модель пунктуальных схем Гильберта поверхности // Тр. МИАН. 1995. Т. 208. С. 318-334.

[7] Фултон У. Теория пересечений. М.: Мир, 1989.

[8] Шафаревич И. Р. Основы алгебраической геометрии. М.: Наука, 1988.

Ярославский государственный педагогический университет им. К. Д. Ушинского 